\title{
Premiers résultats d'une enquête sur la Trichinose des animaux sauvages en France
} (1976-1977)

\author{
par M. ARTOIS \\ (Collaboration technique: A. Magnin, Y. Gérard et J. George) \\ Ministère de l'Agriculture, Direction de la Qualité, Services Vétérinaires, \\ Centre National d'Etudes sur la Rage, B.P. $n^{\circ}$ 9, F 54200 Malzeville.
}

\section{Résumé.}

201 renards (Vulpes vulpes), 18 rats surmulots (Rattus norvegicus), 1 rat musqué (Ondatra zibethica), 1 écureuil (Sciurus vulgaris), 1 mulot (Apodemus sp.), 8 lièvres (Lepus capensis), 3 lapins de garenne (Oryctolagus cuniculus), 2 sangliers (Sus scrofa), 4 chevreuils (Capreolus capreolus), 1 chamois (Rupicapra rupicapra) et 3 rennes (d'élevage) (Rangifer tarandus) ont été analysés par digestion pepsique pour recherche de Trichinose. 8 renards originaires de l'Est et du Sud-Est de la France ont été trouvés infestés par ce parasite.

\section{Summary.}

First results of an investigation on trichinosis in Wildlife in France (1976-1977).

201 red foxes, 18 brown rats, 1 muskrat, 1 red squirrel, 1 wood mouse, 8 hares, 3 rabbits, 2 wild boars, 4 roe deers, 1 chamois, 3 reindeers (for farming) were examined by peptic digestion to detect trichinosis. Eight Foxes captured in the east and south-east of France were found infested by this parasite.

Accepté le 8 mai 1978. 
En 1976, l'Administration Centrale à la Direction de la Qualité, chargeait le Centre National d'Etudes sur la Rage d'entreprendre une recherche de la Trichinose sur les animaux sauvages expédiés à Nancy pour diagnostic de rage. Cette note rapporte les premiers résultats obtenus en 1976 et 1977.

Un foyer récent de Trichinose humaine, attribué à la consommation de viande d'un cheval importé, rappelait en France l'existence de cette maladie. Ce foyer concernait une cinquantaine de personnes de la région parisienne en janvier 1976 (Motyka, 1976). Un autre foyer, lui aussi rapporté à la consommation de viande de cheval importé, a été signalé en Italie en septembre 1975 (Mantovani et al. 1976).

Enfin, dernièrement, un foyer familial sans conséquences médicales graves était signalé (Solignac et al. 1977). La famille s'était contaminée en consommant la viande d'un sanglier abattu en Cerdagne. Ce cas est, à notre connaissance, la première contamination humaine à Trichinella spiralis par un animal sauvage en France. L'infestation des animaux sauvages a toujours été soupçonnée en France et révélée pour la première fois sur une belette (Mustela nivalis) et des micro-mammifères en Bourgogne-Franche-Comté (Lancastre et al. 1973).

Signalée sur de nombreuses espèces sauvages dans le monde entier (Euzéby, 1961), l'infestation à Trichinella spiralis a été mise en évidence sur le renard et quelques autres espèces, en particulier : en Allemagne (Schoop und Lamina, 1962), en Autriche (Hinaidy, 1971), en Grande-Bretagne (Beresford-Jones, 1961 et Oldham and Beresford-Jones, 1957), en Italie (Leinati et Marazza, 1958), en Suisse (Horning, 1977).

\section{Matériel et méthode}

Nous prélevons en salle d'autopsie, avant ouverture des crânes, les deux masséters, si nous ne disposons que de la tête, et le diaphragme chaque fois que cela est possible. Depuis que plusieurs cas ont été révélés dans le Jura, la Direction des Services vétérinaires de ce département nous envoie des diaphragmes d'animaux gibiers prélevés spécialement pour cette étude.

Les muscles sont conservés au réfrigérateur à $+4{ }^{\circ} \mathrm{C}$, sans adjonction de conservateur jusqu'à leur examen. Nous réalisons la digestion pepsique artificielle de lots groupés d'une dizaine de prélèvements de $5 \mathrm{~g}$ de muscle chacun. Chaque lot est placé dans un entonnoir dont l'extrémité est reliée à un tube qui recueille les larves libérées par la digestion. L'ensemble est placé à l'étuve à $39^{\circ}$ pendant une vingtaine d'heures. Le contenu du tube est ensuite observé à la loupe binoculaire. Si le lot est positif nous renouvelons la digestion artificielle de chacun des prélèvements du lot.

Depuis peu, le laboratoire dispose d'un trichinoscope, ainsi un fragment d'un gramme de muscle infesté est examiné pour permettre le comptage des kystes.

Enfin nous entretenons sur des rats et des souris de laboratoire une souche de Trichinella spiralis pour permettre le contrôle périodique de la validité de notre technique. 


\section{Résultats}

243 prélèvements ont été examinés à l'aide de la technique de digestion en entonnoir : 201 renards, 18 rats surmulots et 24 animaux d'espèces diverses. 8 renards ont été reconnus infestés et un neuvième n'a pu être confirmé par le trichinoscope en raison d'une insuffisance de muscle.

Voici l'énumération des résultats :

Renards positifs : 1/42 dans l'Ain, 0/5 dans l'Aisne, 1/3 dans la Côte-d'Or, $0 / 4$ dans le Doubs, $1 / 2$ dans l'Isère, 4/32 dans le Jura, $0 / 1$ dans les Landes et le Maine-et-Loire, $0 / 15$ en Meurthe-et-Moselle, 0/9 en Meuse, $0 / 3$ en Moselle, 0/10 dans la Nièvre, 0/2 dans l'Oise, 0/34 dans le Haut-Rhin, 0/1 dans la Haute-Saône, la Saône-et-Loire et la Savoie, 1/2 en Haute-Savoie (le deuxième renard a été trouvé porteur d'une larve pour $5 \mathrm{~g}$ de muscle digéré mais aucun examen supplémentaire n'a pu être fait par manque de muscle, $0 / 25$ dans les Vosges et enfin $0 / 9$ dans le Territoire-de-Belfort.

Par ailleurs les animaux suivants ont donné un résultat négatif: rats surmulots: 2 dans le Jura, 14 en Meurthe-et-Moselle, 1 en Saône-et-Loire et 1 dans les Vosges. 1 rat musqué (Cher), 1 écureuil (Ain), 1 mulot (Meurthe-et-Moselle) et 8 lièvres, 3 lapins, 2 sangliers, 4 chevreuils, 1 chamois et 3 rennes d'élevage dans le Jura.

La répartition des cas positifs (fig. 1) semble indiquer l'existence d'un foyer dans l'Est-Sud-Est du pays. Enfin, la comparaison des comptages des larves à la loupe binoculaire et au trichinoscope montre qu'une importante proportion de larves n'est pas retrouvée dans le tube embouché à l'entonnoir. Aussi, de faibles taux d'infestation (de l'ordre d'une larve par gramme ou moins) pourraient passer inaperçus.

La poursuite de notre étude précisera l'extension de ce foyer ; par ailleurs outre le renard, d'autres espèces seront examinées en plus grand nombre.

\section{REMERCIEMENTS}

Nous tenons à remercier l'ensemble du personnel du Centre National d'Etudes sur la Rage sans la collaboration duquel ce travail ne pourrait se poursuivre et J.-P. George pour la carte des cas positifs. Nous remercions les $\mathrm{D}^{\mathrm{r}}$ Chevrier et Soulé, du Laboratoire Central de Recherches Vétérinaires de Maisons-Alfort qui ont bien voulu nous permettre d'apprendre et d'utiliser la technique de digestion pepsique en entonnoir et qui nous ont confié la souche de Trichine qui est entretenue au Centre. Enfin, nous remercions M. le Directeur des Services Vétérinaires du Jura qui nous fait parvenir régulièrement des prélèvements pour analyse. 


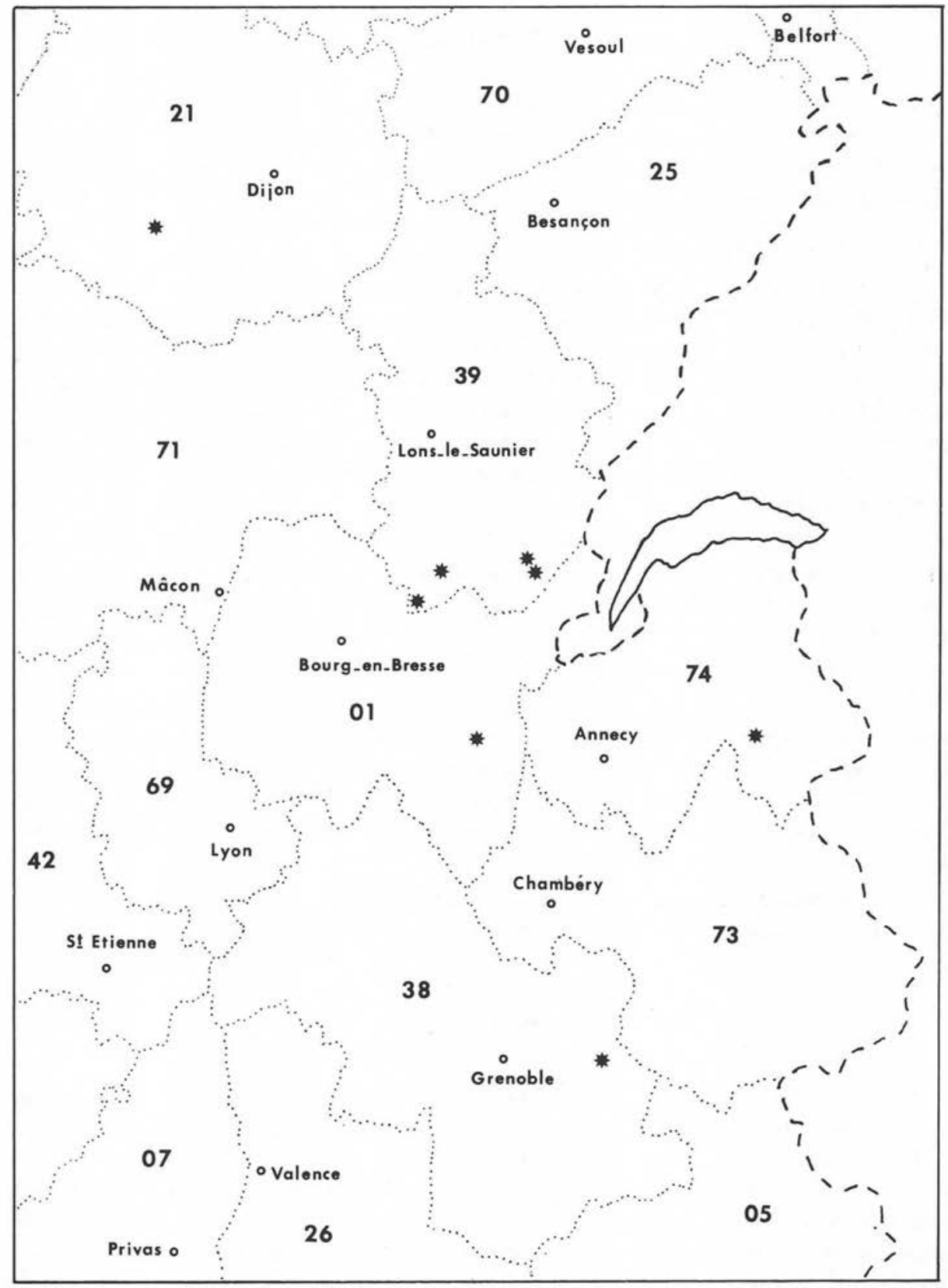

Fig. 1. Répartition géographique des cas positifs (*). 


\section{Bibliographie}

Beresford-Jones W.P. (1961): Observations on the helminths of british wild red foxes. Vet. Rec, $73,882-883$.

Euzéby J. (1961): Trichinose. In «les maladies vermineuses des animaux domestiques», Tome I, Fascicule I, Vigot Frères, édit., Paris, 473 p.

Euzéby J. (1958): Diagnostic expérimental post-mortem direct de la Trichinose. In «Diagnostic expérimental des Helminthoses animales », Vigot Frères, édit., Paris, 367 p.

Hinaidy H. K. (1971) : Die Parasiten Fauna des Rotfuchses, Vulpes vulpes (L.), in Osterreich. Zbl. Vet. Med. B, 18, 21-32.

Horning B. (1977): Weitere Trichinenfunde in der Schweiz (1975-1976). Schweiz. Arch. Tierheilk, $119,337-339$.

Lancastre F., Houin R., Campana-Rouget Y., Le Fichoux Y., Deniau M. (1973): Découverte en France dans la région de Bourgogne-Franche-Comté, d'un foyer de trichinose sauvage. Ann. Parasitol. Hum. Comp., 48, 315-317.

Leinati L., Marazza V. (1958) : La trichinosi negli animali Selvatici in Italia. Atti. Soc. Ital. Scienze Vet., 12, 351-360.

Mantovani A., Filippini I., Saccheti A., Bergomi S., Cavrini C., Marastroni G., Balestrazzi V., Baldelli R., Battelli G., Canestri-Trotti G., Gaiardi S., Mari S., Sanguinetti V., Ambrosi M., Polidori G. A., Virgili-Fioretti D. (1976): Observations sur un foyer de Trichinose humaine en Italie. Bull. Acad. Vet. Fr., 49, 213-217.

Motykas S. (1976) : Enquête épidémiologique à propos d'un foyer de trichinose humaine dans la région parisienne. Bull. Acad. Vet. Fr., 49, 95-99.

Oldham J. N., Beresford-Jones W.P. (1957): Trichinella spiralis in the wild red fox in England. $B r$. Veterinary J., 113, 34-35.

Schoop G., Lamina J. (1962) : Die Rolle des Fuchses in der Epidemiologie der Trichinosis. Deutsche Med. Wochenschr., 87, 335-339.

Solignac H., Gradvohl P., Camo J., Selles R. (1977): Trichinose familiale après consommation de sanglier. Nouv. Presse Med., 6, 2995-2996. 